\title{
Investigation of the accuracy of details positioning in devices with elastic grip pawls
}

\author{
Angel Lengerov \\ Technical University -Sofia, Plovdiv Branch \\ Plovdiv, Bulgaria \\ anlen@tu-plovdiv.bg
}

\begin{abstract}
This paper presents the results of a study of the accuracy of fixing body details on a flat surface and two elastic grip pawls. The obtained experimental results, concerning the displacement of a detail in longitudinal and transverse direction, have been compared with the analytically obtained errors, resulting from positioning a detail on both a cylindrical and a sheared grip pawl. It has been found that the results for the accuracy of details positioning in devices with elastic grip pawls are better than those, related to using a sheared grip pawl and a cylindrical one.
\end{abstract}

Keywords - accuracy, elastic grip pawls, device

\section{INTRODUCTION}

The fixtures are a work-holding or support devices used in the manufacturing industry. They are used to accurately locate and position the workpiece in a specific location and orientation and support it during machining, with respect to the tool in order to maintain the specified tolerances under the cutting and clamping forces [5].

The devices used by CNC machines should not limit the technological capabilities and flexibility of the machines, which defines some basic requirements for them as [2]: the need for a complete orientation of the part in the fixture, because the dimensions are obtained automatically by CNC machining systems; Need for complete orientation of the device and parallelism of its coordinate axes with those of the machine.

Fixtures used in CNC machines are classified as general purpose, modular, flexible or reconfigurable workholders. There has been significant research on automated flexible, reconfigurable and modular fixtures design [1]. Engineering analysis methods such as kinematic analysis, finite element analysis and expert systems are often used in the CAFD. Expert systems have been developed for assisting the fixture design and selection. They are built on rule based systems, which consist, synthesizing rules, constraining rules and strategic rules [4].

The location of small and medium-sized prismatic parts is often done on a flat surface and two pins, one of which is made cylindrical and the other is cut [2]. This scheme has become necessary due to its significant advantages: simple construction of the fixture, the ability to comply with the principle of unity of the bases, the accessible a approach direction of the cutting tool from different sides of the workpiece and more. But it also has a significant disadvantage, which is emphasized in the following particular case: with a small diameter of the holes, the distance between the center of holes and pins must be made with particularly high accuracy.

To eliminate these defects, a method has been proposed for positioning prismatic parts on a flat surface in two elastic pins. The two base pins are cylindrical and assemble the holes in a transition joint. The error of distance between the center of the holes is compensated by the elastic deformation of the console part of the pins, which has a reduced cross section.

The aim of the present work is to investigate the accuracy of locating prismatic details in a fixture with elastic pins. The obtained results can be used in the design of fixtures, as well as in the development of expert systems for CAFD.

\section{MEthodOLOGY}

The experimental setup (system) of the study is shown in Fig.1. The error of the position of the part when basing it in the fixture is determined in the fixed to the workpiece, coordinate system XYZ. Control points on the surface of the part, through which the error of the establishment in space can be registered, are selected according to the principle the 3-2-1 locating principle (6 points location) [2, 5 , 6]. Their position is chosen depending on the 
configuration of the part and at the maximum possible distance between them.

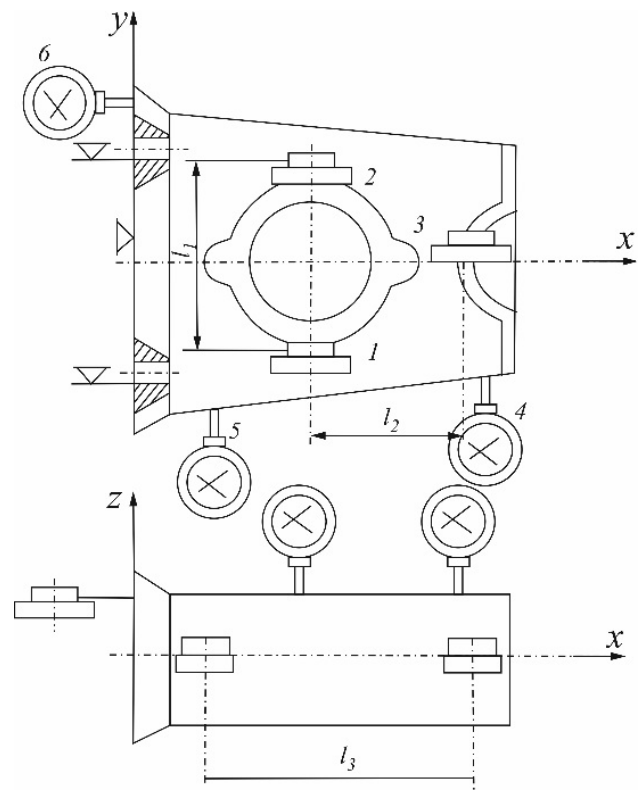

Fig. 1. The experimental setup

Points 1, 2 and 3 determine the position of the part in the plane $\mathrm{xOy}$ and rotation relative to the $\mathrm{x}$ and $\mathrm{y}$ axes, and points 4 and 5 - the movement along the y-axis and rotation relative to the $\mathrm{z}$-axis. The sixth point determines the movement of the part along the $\mathrm{x}$-axis. The error of the position of the part at each of these points is reported by means of measuring clocks with a division value of 0,001 $\mathrm{mm}$, fixed to the mass of the machine by means of magnetic holder.

\section{RESULTS AND DISCUSSION}

100 attempts were performed for three details with an error of the distance between the centres of holes of 0 $\mathrm{mm},-0,25 \mathrm{~mm}$ and $+0,25 \mathrm{~mm}$.

The rotations relative to the three coordinate axes were calculated for each experiment using the equations:

$$
\begin{aligned}
\alpha_{x} & =\frac{\Delta_{1}-\Delta_{2}}{l_{1}}, \\
\alpha_{y} & =\frac{\frac{\Delta_{1}+\Delta_{2}}{2}-\Delta_{3}}{l_{2}}, \\
\alpha_{z} & =\frac{\Delta_{4}-\Delta_{5}}{l_{3}},
\end{aligned}
$$

where $\alpha_{x}, \alpha_{y}, \alpha_{z}$ are the angular deviations from the three coordinate axes;

$\Delta_{i}$ - the measured deviation of the respective clock;

$l_{1}, l_{2}, l_{3}$ - the distances between the clocks, according to fig.1.

The obtained results are statistically processed and are shown in Table. 1 and graphically in Fig. 2 and Fig. 3.
TABLE 1

\begin{tabular}{|c|c|c|c|c|c|c|}
\hline \multirow{2}{*}{$\begin{array}{c}\text { Error in } \\
\text { the center- } \\
\text { to-center } \\
\text { distance }\end{array}$} & $\begin{array}{c}\text { Error } \\
\text { character- } \\
\text { ristics }\end{array}$ & $\begin{array}{c}\alpha_{\mathrm{x}}, \\
\mathrm{s}\end{array}$ & $\begin{array}{c}\alpha_{\mathrm{y}}, \\
\mathrm{s}\end{array}$ & $\begin{array}{c}\alpha_{\mathrm{z}}, \\
\mathrm{s}\end{array}$ & $\begin{array}{c}\text { by the } \\
\text { y axis }\end{array}$ & $\begin{array}{c}\text { by the } \\
\text { x axis }\end{array}$ \\
\cline { 3 - 7 } $0 \mathrm{~mm}$ & $\begin{array}{c}\text { Mean } \\
\text { value }\end{array}$ & 4 & 5 & 3 & 0,7 & 2,3 \\
\cline { 2 - 7 } & Dispersion & 5 & 8 & 8 & 5,8 & 2,7 \\
\cline { 2 - 7 } & $\begin{array}{c}\text { Deviation } \\
\text { zone }\end{array}$ & 19 & 34 & 34 & 25,9 & 11,9 \\
\hline \multirow{3}{*}{$-0,25 \mathrm{~mm}$} & $\begin{array}{c}\text { Mean } \\
\text { value }\end{array}$ & 2 & 6 & 16 & $-1,73$ & $-0,07$ \\
\cline { 2 - 7 } & Dispersion & 8 & 7 & 8 & 7,34 & 1,94 \\
\cline { 2 - 7 } & $\begin{array}{c}\text { Deviation } \\
\text { zone }\end{array}$ & 36 & 29 & 35 & 32,8 & 8,7 \\
\hline & $\begin{array}{c}\text { Mean } \\
\text { value }\end{array}$ & 19 & 15 & 13 & 2,4 & 2,8 \\
\cline { 2 - 7 }$+0,25 \mathrm{~mm}$ & Dispersion & 9 & 5 & 6 & 10,6 & $\mathrm{~V} 7$ \\
\cline { 2 - 7 } & $\begin{array}{c}\text { Deviation } \\
\text { zone }\end{array}$ & 33 & 22 & 3 & 47,4 & 6,4 \\
\hline
\end{tabular}

In Fig. 2 shows the experimental and theoretical curves of the distribution of the obtained error along the y-axis, and Fig. 3 - along the $x$-axis, in case of deviation the distance between the centers of the holes.

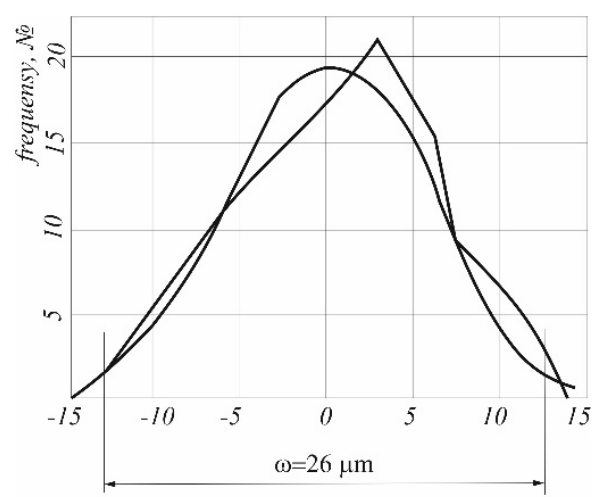

a)

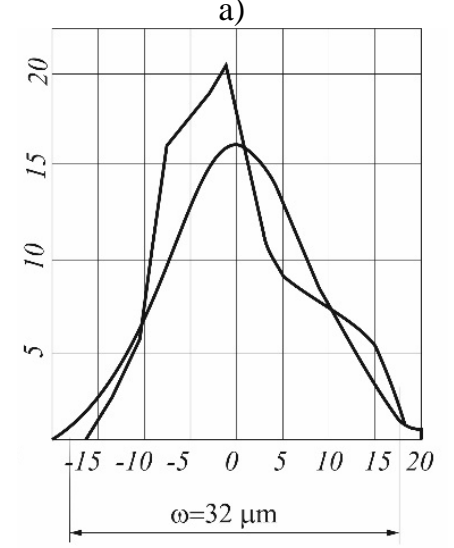

b) 


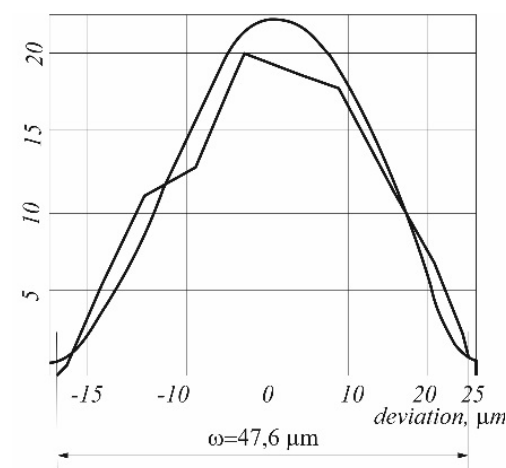

c)

Fig. 2. Error of displacement of the part in the transverse cros direction in case of error of the center distance: a) $0 \mathrm{~mm}$; b) $-0,25 \mathrm{~mm}$; $)+0 \mathrm{~mm}$

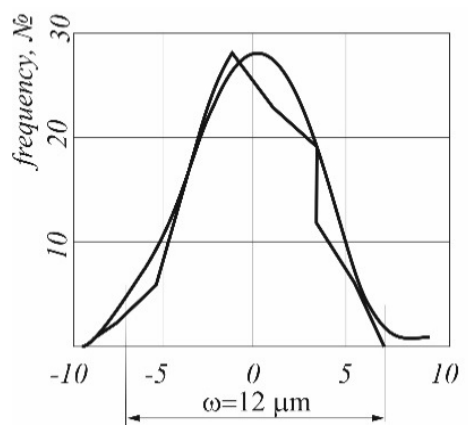

a)

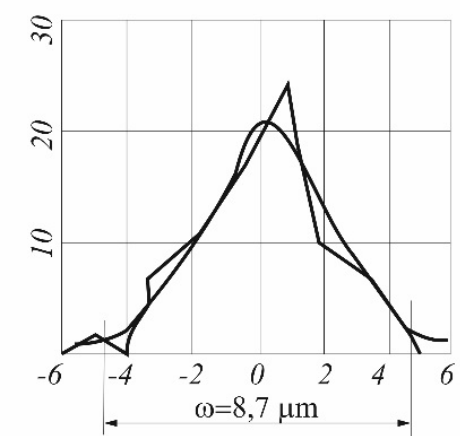

b)

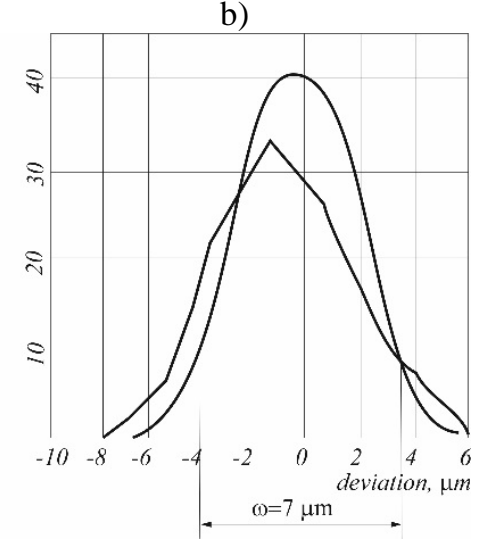

c)

Fig. 3. Error of displacement of the part in the transverse direction in case of error of the center distance: a) $0 \mathrm{~mm}$; b) $-0,25 \mathrm{~mm}$; c) $+0 \mathrm{~mm}$
It can be seen from the figures that there is a good agreement of the experimental curve with the normal distribution law, which is confirmed by Pearson's criterion $\chi^{2}$.

Larger deviations of the center-to-center distance result in larger scattering dispersion fields. This does not contradict the physical meaning of the elastic deformation of the pins.

An estimate of the error obtained by establishing on a flat surface and two elastic pins can be made by comparing it with the error when establishing a cylindrical and cut pin for the same conditions. As no such device is available, this comparison is made analytically.

The width $b$ of the cut pin is determined by the condition [3]:

$$
J_{\min }+\frac{d}{b} J_{\min } \geq T_{L \mathrm{det}}+T_{L \mathrm{dev}}
$$

where $J_{\min }$ - the minimum guaranteed clearance fit between the pins and the holes;

$d$ - diameter of the cut pin;

$b$ - width of the cut pin;

$T_{L \text { det }}$ - tolerance of between the center distance of the holes for the part;

$T_{L \mathrm{dev}}$ - tolerance of between the center of the pins from the device.

For specific conditions, when clearance fit $\phi 8 \frac{H 7}{f 7}$ with a minimum guaranteed clearance $J_{\min }=0,013 \mathrm{~mm}$, when the tolerance of the center-to-center distance $T_{L \mathrm{~d} e v}= \pm 0,02$ according to [3] for the width of the cut pin obtained in $b=0,198 \mathrm{~mm}$. A pin of this width cannot guarantee the normal operation of the device.

To obtain a width $b=2,5 \mathrm{~mm}$, the tolerances of the center-to-center distances must be adjusted accordingly $T_{L \text { det }}= \pm 0,02 \mathrm{~mm}, T_{L \text { dev }}= \pm 0,005 \mathrm{~mm}$, which means that the base holes of the part must be machined on a coordinate borring with an optical device for coordinate reading. Such a solution is unacceptable in the conditions of large-scale and mass production.

With the same tolerances at center distances, a sufficient width of the cut pin can be ensured by increasing the diameter of the holes. For the above conditions, it is obtained $b=3 \mathrm{~mm}$ when clearance fit $\phi 51 \frac{H 7}{f 7}$ the pins with the holes. Such a solution is also impermissible, since the diameter of the holes is determined by the construction of the part.

When establishing a cylindrical and cut pin, the displacement of the workpiece in a direction perpendicular 
to the axis of the pins is determined by the maximum clearance $J_{\max }$ between the pin and the hole according to [3]:

$$
J_{\max }=J_{\min }+\frac{T_{1}}{2}+\frac{T_{1}^{\prime}}{2}+\frac{T_{1 w}}{2}
$$

where $T_{1}$ is the tolerance of the hole diameter;

$T_{1}^{\prime}$ - the tolerance of the diameter of the pin;

$T_{1 w}$ - the wear tolerance of the pin.

At clearance fit $\phi 51 \frac{H 7}{f 7}, J_{\max }=0,075 \mathrm{~mm}$ is obtained.

The study gives $W_{\max }=48 \mu \mathrm{m}$.

The largest angle of rotation when establishing a cylindrical and cut pin can be determined by the relation

$$
\sin \alpha=\frac{J_{\max }+J_{\max }^{\prime}}{L}
$$

where $J_{\max }^{\prime}$ is the clearance between the hole and the cut pin;

$L$ - center distance.

For the specific case we get $\alpha^{\prime}=3^{\prime}$. The study obtained a maximum rotation $\alpha_{x}=36 "$.

\section{CONCLUSIONS}

A comparative study of the error of establishing on a flat surface and two elastic pins and the popular scheme with a cylindrical and cut pins performed in this paper.

The conducted researches show that when establishing on flat surfaces and elastic pins, admissible linear and angular errors of the position of the workpiece are obtained for conditions under which the establishment on the cylindrical cut pin is impossible or inexpedient.

\section{REFERENCES}

[1] V. Ivanov, Process-Oriented Approach to Fixture Design. In Book: Advances in Design, Simulation and Manufacturing (pp. 42-50), 2019.

[2] G. Jordanov, Technological equipment, Sofia, 2014, (In Bulgarian).

[3] I. Zamfirov, Technological equipment, Ruse, 1994, (In Bulgarian).

[4] D. Stephanson, J. Agapiou, Metal cutting theory and praktice, Taylor \& Francis, Inc. 2016.

[5] O. Mihaylov, G. Nikolcheva, An integrated RBR fixture design system, Vol. 5, CBU International Conference Proceedings, 2017.

[6] H. Metev, T. Kuzmanov, K. Krumov, Determining the accuracy taking into account the phenomenon of technological heredity, TUGabrovo, vol.49, pp.22-28, ISSN 1310-6686, 2015, (In Bulgarian). 